\title{
Single Image Defogging Method Based on Adaptive Modified Dark Channel Value
}

\author{
Peiling Li, Feng Wang, Yitao Liang and Xiao Zhang \\ College of Information Science and Engineering, Henan University of Technology, Zhengzhou \\ 450001, China
}

\begin{abstract}
Keywords: Image defogging, Adaptive modification, Dark channel prior, Atmospheric scattering model.
\end{abstract}

\begin{abstract}
In the conventional image defogging algorithm based on dark channel prior, the calculation deviation of dark channel value of image edge position could lead to halo effect on image. In this paper, a method for correcting dark channel value is proposed. Firstly, according to the gray difference between the minimum image and dark channel image, an adaptive threshold is obtained to determine the edge image pixels with drastic depth gray value changes. Secondly, with the threshold, the gray values are actively modified. Finally, clear fog-free image can be restored according to the atmospheric scattering model. The test results of several groups of foggy images show that after the application of the method, the halo effect effectively removed, the image color deviation corrected, and the contrast improved. The restored image looks clearer and more natural.
\end{abstract}

\section{Introduction}

In foggy weather, suspended particles in the atmosphere can scatter light. The reflected light of the object is attenuated, resulting in image contrast reduction, color distortion and detail blurring. By defogging, the clarity and contrast of the scene in the image can be improved. Existing defogging methods are mainly divided into two categories: non-physical model-based method and physical model-based method [1]. The essence of non-physical method [2-4] does not analyze the reasons of image degradation and has a wide range of applications, but it may cause loss of some information of image and distort the image. The essence of physical model-based method is image restoration [5]. By estimating the parameters of the model with known constraints, a clear and natural scene image can be restored eventually. Fattal [6] proposes a priori restoration algorithm for reflectivity. The algorithm solves the distortion problem better, but the processing effect is worse when the color information is less. Wang et al. [7] found that there was a linear relationship between the minimum channel of foggy image and fog free image. An improved method based on quad-tree was proposed to estimate the atmospheric light value. Meng et al. [8] used boundary constraints to roughly estimate transmittance and regularization to optimize transmittance for defogging. He et al. [9] found that there is at least one color channel with very low gray value in the $\mathrm{R}, \mathrm{G}$ and $\mathrm{B}$ color channels of the local region of most color fog-free images. They proposed an image defogging algorithm based on dark channel priori, but the restored image has halo effect. Later, He et al. [10] used guided filtering instead of soft matting to refine the transmittance. The efficiency has been greatly improved, but new problems such as incomplete defogging have arisen [11]. On the basis of literature [9], [12-15] presents improved algorithms, which improve the robustness and real-time performance of the algorithms to some extent, but there are still some shortcomings.

When image fog removal is based on dark channel theory, it is a conventional idea to optimize the transmittance to improve the effect of fog removal. This paper improves the algorithm from dark channel value. It does not need to refine the transmittance, but also restrains the halo effect effectively, and restores the color and contrast of the image quickly. 


\section{Background}

\section{Atmospheric Scattering Model}

McCartney [4] deduced the atmospheric scattering model, as shown in equation (1):

$$
E(d, \lambda)=E_{0}(\lambda) e^{-\beta(\lambda) d}+E_{\infty}(\lambda)\left(1-e^{-\beta(\lambda) d}\right) .
$$

Where $E(d, \lambda)$ is the illumination intensity of the target scene light when it reaches the imaging equipment, $E_{0}(\lambda) e^{-\beta(\lambda) d}$ is the direct attenuation term, which represents the light intensity of the attenuated target scene, $E_{\infty}(\lambda)\left(1-e^{-\beta(\lambda) d}\right)$ is the ambient atmospheric light. Where $\beta(\lambda)$ represents the atmospheric scattering coefficient, $E_{0}(\lambda)$ represents the light intensity of the scene at $d=0$, and $E_{\infty}(\lambda)$ represents the atmospheric light intensity at infinity.

The formula is simplified and applied to digital images: $I(x)=E(d, \lambda), J(x)=E_{0}(\lambda), t(x)=e^{-\beta(\lambda) d}$, $A=E_{\infty}(\lambda)$.The foggy image degradation model is obtained [16]:

$$
I(x)=J(x) t(x)+A(1-t(x)) .
$$

Where $x$ is a single pixel coordinate, $I(x)$ denotes the original foggy image, $J(x)$ denotes the image after fogging, $t(x)$ denotes the transmission, $A$ denotes the atmospheric light value. The known information is only $I(x)$. In order to recover $J(x)$, we need to obtain A and $t(x)$ from $I(x)$. Therefore, we need some auxiliary information or prior knowledge.

\section{Dark Channel Priori Theory}

According to the dark channel priori theory, combined with the foggy image degradation model, $t(x)$ and $A$ can be estimated, and then clear images can be restored. Assuming that the atmospheric light value $A$ is given, and the transmission in a local region $\Omega(x)$ is a fixed value, the transmission in the local region is $\tilde{t}(x)$, and the color channel is $c \in\{r, g, b\}$, the formula (2) first filters the minimum value in the local region, and then calculates the minimum value in three color channels:

$$
\min _{x \in \Omega(x)}\left(\min _{c}\left(I^{c}(x)\right)\right)=\tilde{t}(x) \min _{x \in \Omega(x)}\left(\min _{c}\left(J^{c}(x)\right)\right)+A^{c}(1-\tilde{t}(x)) .
$$

The dark channel intensity of fog-free images is very low near 0 , so there are:

$$
\tilde{t}(x)=1-\min _{y \in \Omega(x)}\left(\min _{c}\left(\frac{I^{c}(x)}{A^{c}}\right)\right) .
$$

In real life, even if the weather is very clear, fog still exists when looking at the distance [17], so the existence of fog can perceive the depth. Therefore, a constant $\omega(0<\omega \leq 1)$ is introduced in the formula to keep a part of the fog pertinently, that is, the formula for calculating transmission is as follows:

$$
\tilde{t}(x)=1-\omega \min _{c}\left(\min _{y \in \Omega(x)}\left(\frac{I^{c}(y)}{A^{c}}\right)\right) .
$$

\section{The Proposed Method}

This paper improves the image defogging algorithm based on dark channel theory. Firstly, the gray value of the edge position in the dark channel image which changes dramatically is corrected, and then the modified dark channel image is filtered by means to smooth the area where the depth of field changes slowly. Finally, the clear image is restored. 


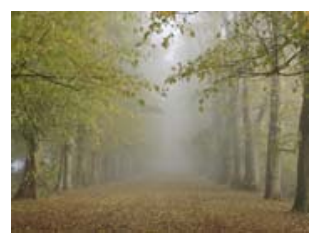

(a)

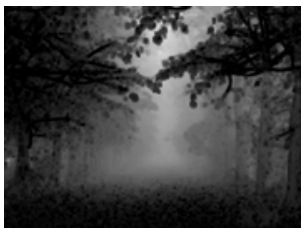

(b)

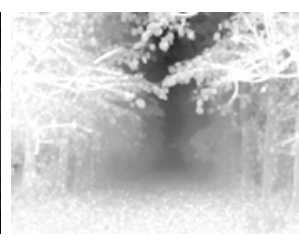

(c)

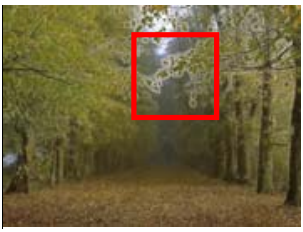

(d)

Figure 1. Effect Charts of Each Step of Dark Channel Algorithms. From left to right, the order ishaze image, dark channel image, transmittance image and Ref.[9]'s restored image.

\section{Adaptive Modification of Dark Channel Value Based on Gray Difference}

According to the dark channel priori theory, under foggy weather conditions, the bigger the depth of field of the object in the image, the more gray-white the color is affected by fog, and the bigger the value of the dark channel is. Conversely, the smaller the dark channel value [17]. Therefore, halo effect is easy to occur in restored images where the depth of field changes dramatically. For example, in Fig. 1, because the position of leaf edge is small due to the dark channel value, the restored image has obvious halo generation, as shown in Fig. 1 (d).

To solve this problem, in formula (3), $\min _{c \in\{r, g, b\}}\left(I^{c}(x)\right)$ is the minimum value graph, denoted as $\operatorname{dark1}(x), \min _{x \in \Omega(x)}\left(\min _{c \in\{r, g, b\}}\left(I^{c}(x)\right)\right)$ is the dark channel graph, denoted as dark2(x), and the adaptive threshold $T$ is obtained according to the gray difference $h(x)$ between the minimum value map and the dark channel image. According to $h(x)$ and $T$, the position of sharp change of depth of field in image is determined, and then the gray value of dark channel is corrected. The steps are as follows:

Step 1: Let the image to be processed be $I(x)$, calculate dark1(x), dark2(x), $h(x)$ and $T$. Among them:

$$
\begin{aligned}
& h(x)=\operatorname{abs}(\operatorname{dark} 1(x)-\operatorname{dark} 2(x)) . \\
& \mathrm{T}=\max (h(x)) / 4 .
\end{aligned}
$$

Step 2: Traversing through $x$ in $h$, when $h(x)$ is less than $T$, it is considered that the pixel is located in the area where the depth of field changes slowly, the dark channel value is correctly calculated, and the value of $\operatorname{dark} 2(x)$ is still taken as the dark channel value; when $h(x)$ is greater than or equal to $T$, it is considered that the pixel is located in the position where the depth of field changes dramatically, and the value of $\operatorname{dark1}(x)$ is taken as the dark channel value. For formula (9):

$$
\operatorname{dark} 3(x)=\left\{\begin{array}{l}
\operatorname{dark} 1(x), h \geq T \\
\operatorname{dark} 2(x), h<T
\end{array} .\right.
$$

Step 3: The $\operatorname{dark3(x)}$ is filtered by means of mean filter, and the improved dark channel image is obtained:

$$
\operatorname{dark}(x)=\underset{x \in \Omega(x)}{\operatorname{mean}}(\operatorname{dark} 3(x)) \text {. }
$$

Step 4: Calculate the transmittance according to formula (5) and restore the fog image according to formula (2).

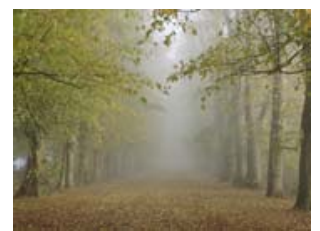

(a)

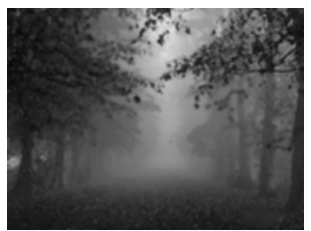

(b)

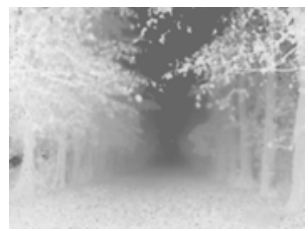

(c)

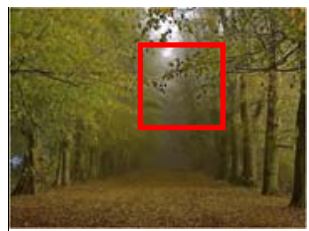

(d)

Figure 2. Effect Charts of Each Step of our Algorithms. From left to right, the order is haze image, dark channel image, transmittance image and our restored image. 
Fig. 2 is the improved dark channel contrast image. It can be seen that the dark channel image obtained by this algorithm has been significantly improved: the application of formula (8) makes the image thinner in the edge part of the field depth which changes dramatically, and the application of formula (9) makes the image smoother in the area where the field depth changes slowly.

\section{Experimental Result}

The simulation software environment is MATLAB R2017a, the operating system is Windows 10, and the computer hardware is configured as Intel (R) Core (TM) i7-8550U CPU @ 1.80GHz and 8GB RAM.

\section{Subjective Evaluation}

In order to prove the advantages of this algorithm, the representative literatures [8-10] in the field of image defogging are selected and compared with the algorithm in this paper. The results are shown in Fig. 3. Through observation, it can be found that the image of document [8] has a serious color deviation for the image containing a large area of sky, (b3) the sky region is black, (c3) the sky region color is somewhat supersaturated; the image details and contrast after document [9] processing remain good, but there are obvious halo effects, as shown in (a4) and (c4); and document [10] uses guided filtering fine. Transmittance eliminates halo effect, but fog still exists in the image after fogging removal, as shown in (a2) and (c2). Compared with the results of the literature method, the details, color and contrast of the image processed by the algorithm are better preserved for the images with different sky area and depth of field. (a5) The details of the leaves are kept intact while the fog is removed thoroughly; (b5) the sky area is more in harmony with the building. (c5) In the near-range part of the image, fog removal is more thorough, and the color of the far-range sky region is more consistent with the original image. (d5) image color is more natural.

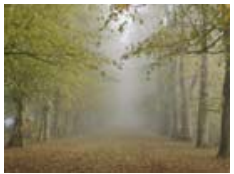

(a1)

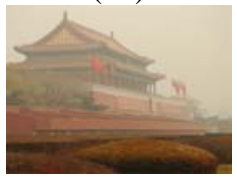

(b1)

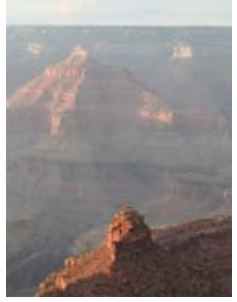

(c1)

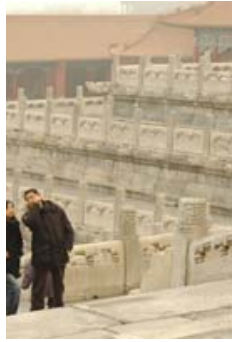

(d1)

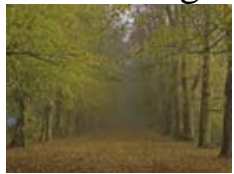

(a2)

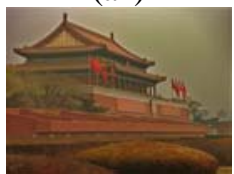

(b2)

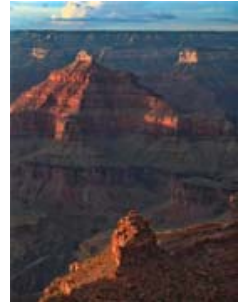

(c2)

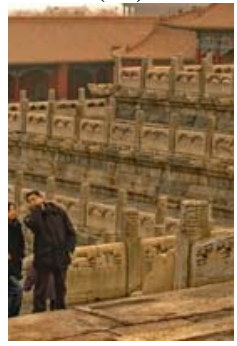

(d2)

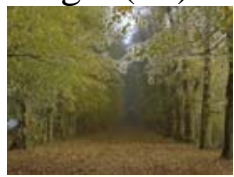

(a3)

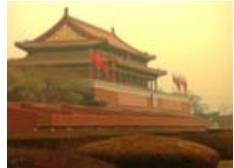

(b3)

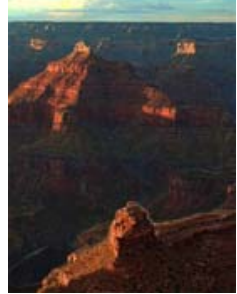

(c3)

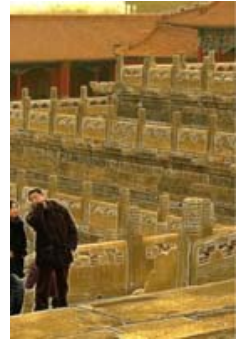

(d3)

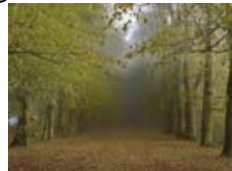

(a4)

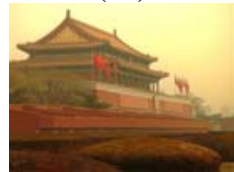

(b4)

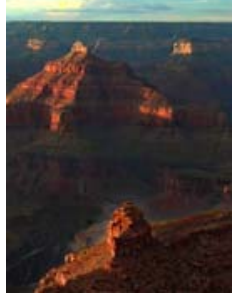

(c4)

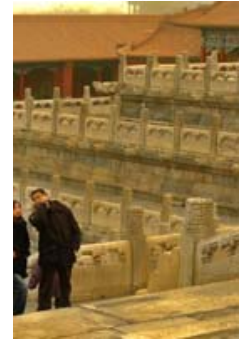

(d4)

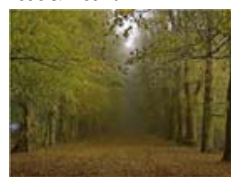

(a5)

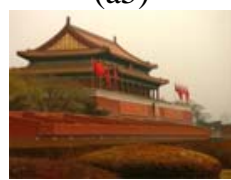

(b5)

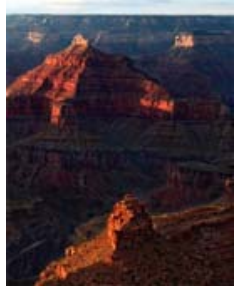

(c5)

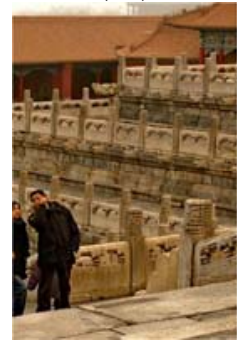

(d5)

Ref.[8]'s method

Ref.[10]'s method

Figure 3. Comparison of restoration results of each algorithm. The first column shows a foggy image. The second to fourth columns show the restored images obtained by the literature [8-10] algorithm. The last column is the restored image obtained by our method. 


\section{Objective Evaluation}

In order to objectively compare the de-fogging effects of the algorithms in this paper with those in literature [8-10], three parameters of information entropy, average gradient and contrast are used as the evaluation indexes of image de-fogging effect.

Table 1. Objective quality evaluation of restored images based on different algorithms

\begin{tabular}{|c|c|c|c|c|c|c|c|c|c|c|c|c|}
\hline \multirow[b]{2}{*}{ Images } & \multicolumn{4}{|c|}{ Information Entropy } & \multicolumn{4}{|c|}{ Average Gradient } & \multicolumn{4}{|c|}{ Image Contrast } \\
\hline & $\begin{array}{c}\text { Meng et } \\
\text { al. [8] }\end{array}$ & $\begin{array}{c}\text { He et al. } \\
{[9]}\end{array}$ & $\begin{array}{c}\text { He et al. } \\
{[10]}\end{array}$ & $\begin{array}{c}\text { Our } \\
\text { method }\end{array}$ & $\begin{array}{c}\text { Meng et } \\
\text { al. [8] }\end{array}$ & $\begin{array}{c}\text { He et al. } \\
\text { [9] }\end{array}$ & $\begin{array}{c}\text { He et al. } \\
{[10]}\end{array}$ & $\begin{array}{c}\text { Our } \\
\text { method }\end{array}$ & $\begin{array}{l}\text { Meng et al. } \\
\text { [8] }\end{array}$ & $\begin{array}{c}\text { He et al. } \\
\text { [9] }\end{array}$ & $\begin{array}{c}\text { He et al. } \\
{[10]}\end{array}$ & $\begin{array}{c}\text { Our } \\
\text { method }\end{array}$ \\
\hline Fig,a & 7.1753 & 7.3748 & 7.3624 & 7.4476 & 0.0235 & 0.0237 & 0.0222 & 0.0269 & 17.7153 & 24.3097 & 23.8157 & 24.8568 \\
\hline Fig,b & 7.3741 & 7.4349 & 7.4456 & 7.7943 & 0.0186 & 0.0170 & 0.0160 & 0.0231 & 29.1569 & 68.8704 & 68.6940 & 70.7983 \\
\hline Fig,c & 6.7695 & 6.8663 & 6.8122 & 6.8762 & 0.0170 & 0.0188 & 0.0160 & 0.0189 & 23.5897 & 31.8071 & 31.2893 & 37.1755 \\
\hline Fig,d & 7.5700 & 7.7440 & 7.7154 & 7.7707 & 0.0365 & 0.0409 & 0.0308 & 0.0441 & 28.0578 & 32.5174 & 30.8666 & 39.9106 \\
\hline
\end{tabular}

The objective quality evaluation of image restored by each algorithm in Fig. 3 is shown in Table 1. Compared with literature [8-10], the image restored by this algorithm has higher values of information entropy, average gradient and contrast. This shows that the image restored by this algorithm retains more detail information while improving contrast, which is consistent with the results of subjective observation.

\section{Conclusion}

In this paper, aiming at the reason of halo effect when reconstructing fog image based on dark channel method, an adaptive threshold method based on gray difference is proposed to improve dark channel image. The experimental results show that the algorithm can eliminate halo effect without optimizing the transmittance. It can maintain more image details while improving image contrast, and better restore foggy images with close, long and complex depth of field.

\section{Acknowledgement}

This research was sponsored by the National Natural Science Foundation of China (No. 61705060), the Natural Science Project of Henan Science and Technology Department (No. 182300410036), and the Key Scientific Research Program of Henan University (No. 17A510008).

\section{References}

[1] Yu Jing, Xu Dongbing, Liao Qingmin, Image defogging: a survey, J. Journal of Image and Graphics. 2011, 16(9): 1561-1576.

[2] Kim J Y, Kim L S, Hwang S H, An advanced contrast enhancement using partially overlapped sub-block histogram equalization[C]// IEEE International Symposium on Circuits and Systems. 2000. Proceedings. ISCAS. IEEE, 2002:537-540 vol.4.

[3] Stark J A, Adaptive image contrast enhancement using generalizations of histogram equalization, J. IEEE Transactions on Image Processing. 2002, 9(5):889-896.

[4] Wang W, Xu L, Retinex Algorithm on Changing Scales for Haze Removal with Depth Map, J. International Journal of Hybrid Information Technology. 2014, 7(4):353-364.

[5] MCCARTNEY E J, Optics of the atmosphere: scattering by molecules and particles, J. John Wiley and Sons. 1976, 12(5):123-129.

[6] FATTAL R, Single image dehazing, J. // ACM Transactions on Graphics, SIGGRAPH, 2008, 27(3): $1-9$

[7] Wang W, Yuan X, Wu X, et al, Fast Image Dehazing Method Based on Linear Transformation, J. IEEE Transactions on Multimedia. 2017, 19(5):1142-1155 
[8] Meng G , Wang Y , Duan J , et al, Efficient image dehazing with boundary constraint and contextual regularization, C. // 2013 IEEE International Conference on Computer Vision. IEEE, 2014.

[9] He K, Sun J, Tang X, Single image haze removal using dark channel prior, C. // Computer Vision and Pattern Recognition, 2009. CVPR 2009. IEEE Conference on. IEEE, 2009:1956-1963.

[10]He K, Sun J , Tang X, Guided Image Filtering, J. IEEE Transactions on Pattern Analysis \& Machine Intelligence. 2013, 35(6):1397-1409.

[11] Riaz I, Yu T , Shin H , et al, Real-time single image dehazing using block-to-pixel interpolation and adaptive dark channel prior, J. IET Image Processing. 2015, 9(9):725-734.

[12]Ji Xiaoqiang, DAai Ming, Sun Lina, et al. Research on the image haze-removal algorithm based on the prior dark-channel[J]. Journal of Optoelectronics•Laser, 2011, 22(6):926-930.

[13]Xiao Jinsheng, Gao Wei, Zou Baiyu, et al, Image Dehazing Based on Sky-Constrained Dark Channel Prior, J. Acta Electronica Sinica. 2017,45(2):346-352.

[14]Zeng Jiexian, Yu Yonglong, Image defogging and edge preserving algorithm based on dark channel prior and bilateral filtering, J. Journal of Image and Graphics. 2018, 22(2):147-153.

[15]Liu Guo, Lv Qunbo, Liu Yang-yang, Single Image Dehazing Algorithm Based on Adaptive Dark Channel Prior, J. Acta Photonica Sinica. 2018,47(2):179-186.

[16]Chen Lu, He Hongjie, Chen Fan, A Fast Image Defogging Algorithm Based on Edge-maximum Filter, J. Acta Photonica Sinica. 2014,43(11):114-119

[17]Goldstein E B, Blackwell Handbook of Sensation and Perception[M]// Blackwell handbook of sensation and perception. 2005.

[18]Chen Shuzhen, Ren Zhanguang, Lian Qiusheng, Single Image Dehazing Algorithm Based on Improved Dark Channel Prior and Guided Filter, J. Acta Automatica Sinica. 2016, 42(3):455-465. 